\title{
Better Together - A Day Hospital's move towards integrated care
}

*Dr Michele Board Principal Academic Nursing Older People FHSS Bournemouth University Lisa Pigott Day Hospital Manager Christchurch Day Hospital RBCH NHS Foundation Trust Heather Olive Senior Occupational Therapist Christchurch Day Hospital RBCH NHS Foundation Trust

Dr Vanessa Heaslip Principal Academic Nursing FHSS Bournemouth University

\section{Contact details}

* Lead Author

Faculty of Health and Social Sciences Bournemouth University, Christchurch Road, Bournemouth BH1 3LH

mboard@bournemouth.ac.uk Tel 01202961786

\section{Introduction}

As a global society people are living longer; the World Health Organisation (2015) identifies that by 2050 the proportion of the world's population over the age of 60 years will equate to $22 \%$ and the pace of ageing is growing at a rapid rate. This growth in an ageing population is mirrored within the United Kingdom (UK) which has the largest percentage of growth in those aged over 85 years (Office of National Statistic (ONS) 2015a). By 2030, one in five people in England will be over 65 and at that age, men will on average live until 88 and women until 91 years. This is a fact worth celebrating and represents success in society and for modern health care. It has to be remembered that most people report high levels of happiness, health and wellbeing and evidence by Department for Environment, Food and Rural Affairs (DEFRA 2011) that identifies that only half of people over 80 say they live with life limiting long-term conditions. However, despite this "upside" of population ageing, we need to be realistic about the implications for health and care services. We know that as people age they are progressively likely to experience multiple long-term conditions (average of three for people aged over 75 years), including common age-related conditions such as dementia, bone fragility, heart failure and incontinence, frailty, and disability in the 
form of impaired mobility, visual or hearing impairment (Oliver et al. 2014). With an increase in the numbers of older people globally there is also an associated increase in the numbers of people living with complex chronic illness. This has resulted in all countries facing challenges in ensuring that their health and social care systems are ready to address the needs of an ageing society (WHO 2015).

Within the UK the National Health Service (NHS) has been designed around a disease focused service and not complexity or multi-pathology (Roland and Paddison 2013). However a core part of the vision in the NHS five year forward view is a fundamentally different role for acute hospitals (NHS 2014). The needs arising from the shifting population increases the demand on acute hospitals, and they will not be able to meet these challenges by working alone. This changing landscape was seen as an ideal opportunity for a day hospital, in an acute NHS UK Trust to redevelop their service to more effectively meet the needs of their local community. Day Hospitals, originated in the United Kingdom in the 1950s, and in the main they provide an outpatient service as part of an acute hospital trust. A recent Cochrane review (Brown et al. 2015) highlights the existence of Day Hospital across the EU and the USA. Aliberti et al (2016) have also seen the potential of developing day hospital services in Brazil and considered the valuable role this service can provide in meeting the needs of an ageing population. Within the geographical area where the day hospital being discussed in this paper is located, $31.4 \%$ of the population is aged over 65 years (ONS 2015b); as such the patients that present to the service invariably have complex needs and a degree of frailty. As NHS patients they do not pay of the service. Patients attend the day hospital either live in an urban or semi-rural location and access the Hospital in their own privately paid transport or Hospital Transport.

Within the UK there is an increasing drive to identify and manage frailty to avoid unnecessary hospital admissions and if admitted, a reduced length of stay (National Institute Clinical Excellence, (NICE) 2015, British Geriatric Society (BGS) 2014). The gold standard for the assessment of frailty is the Comprehensive Geriatric Assessment (CGA) which includes a multi professional assessment of the patient's needs (BGS 2014). Day hospitals are often staffed by interdisciplinary teams and therefore we argue are ideally placed to undertake the CGA of the frail older population and initiate a comprehensive plan 
of action over a six week period to address the individual person's needs. Interventions that could be initiated include balance exercises, gait re-education, cognitive assessments, reviews of functional safety/independence, blood pressure monitoring and continence management all of which enable a holistic assessment of the patient.

Despite the wealth of expertise within day hospitals, there has been a national decline of this service (Parker et al. 2009). Brown et al. (2015) has argued that further research is needed into the effectiveness of day hospitals and this research should incorporate the views of the patients. In light of this, the day hospital wished to systematically examine their service to identify ways it could be improved to provide a better quality of care to their local community. With this in mind they engaged with a local higher education institute (HEI) that provided Practice Development Unit accreditation (PDU). Practice development is a broad term that covers a range of approaches to health and social care improvement work. It is essentially patient/client focused and uses processes based on teamwork, collaboration and cultural change (McCormack et al 2013). Benefits of practice development are varied and for staff include better communication and team structure (Martin et al 2014), and increased job satisfaction and reduction in staff absence from sickness (Hennessey \& Fry 2016); benefits to patients include higher standards of cleanliness, privacy and dignity, decrease in falls, and pressure sores as well as a decrease in length of hospital stays (Hennessey \& Fry 2016). The HEI PDU process is an 18 month journey in which teams seeking accreditation work with an academic facilitator from the university and at the end are assessed against the nine PDU standards (see table 1). The PDU standards enable the team to work towards developing their practice both for the benefit of their own job satisfaction, but significantly the needs of patients. This process reflects how McCormack et al., (2004, p. 316) defined practice development which is:

"A continuous process of improvement towards increased effectiveness in patient centred care. This is brought about by enabling health care teams to develop their knowledge and skills and to transform the culture and context of care. It is enabled and supported by facilitators committed to systematic, rigorous continuous processes of emancipatory change that reflect the perspectives of both service users and service providers".

As the day hospital worked towards PDU status they were facilitated by an academic from the university, a member of the hospital board as well as key leaders within the department. 
Each Practice Development Unit must demonstrate:

1. Information on the component parts of the integrated team, its members and a description of the health and social care provided by the team

2. An understanding of the service user needs and clear mechanisms for communicating and listening to users and carers

3. A clear action plan that outlines the aims for development of services and team members that has clear links to national and local host organisations priorities

4. A clear leadership structure that supports the delivery and development of multiagency inter professional care in line with the action plan

5. A clearly defined communication structure to ensure the collaboration of team members and the provision of effective inter professional care

6. A clear understanding of the users journey and understanding of the team members contribution to the success of the user experience

7. How services have been developed taking a 'whole systems' approach, ensuring the entire user journey is addressed

8. How developments are clearly researched, evidence based and evaluated and disseminated to demonstrate best practice and the sharing of findings to contribute to the local, national and international evidence base

9. Clear partnerships with an academic department and other agencies required to support research and the dissemination of best practice.

This paper shall now continue to explore examples of how the interdisciplinary team at the Day Hospital came together to redevelop their service providing a better quality of care to the patients, as well as exploring the experiences of staff.

\section{Better Together - working towards PDU status}

The Chartered Institute of Personnel and Development (2012) highlight two integral aspects of any public sector change; articulating a vision and involving staff in creating the vision. T 
At the start of the PDU process the staff spent time thinking about the philosophy of the unit and the unique benefits of the service they could provide. They identified the catch phrase 'better together' which summarised to them their collective strength as a multi professional service. There were no additional funds available to implement changes so staff were encouraged to be creative when considering how the service could be developed. One of the first activities of the day hospital was the review of the patient pathway which was achieved by mapping out the patient journey from referral to discharge which was undertaken by the PDU leadership team, then they asked all of the staff working in the day hospital (administrative, clinical, housekeeping, and volunteers) to contribute their improvement ideas which they referred to as 'light bulb moments'. This generated over 100 ideas all of which were embedded in their PDU project plan (an example of this included changing the time of the routine fire alarm practice to avoid the time when the patients arrived at the day hospital). Each idea represented a meaningful change either directly for patients or for service provision. This process engaged the whole team and ensured that all members of staff felt empowered in the PDU process and felt that they have a voice in the redesign of their service which is fundamental in ensuring staff buy in to change.

\section{Better Together - CGA and fusion of staff roles}

All staff in the department joined different working groups focussing upon different projects to improve service delivery. One such group was the development of staff roles. Many of the patients using the service were frail and the CGA was routinely completed with each member of the interdisciplinary team assessing each patient. Seeing this from the patients' perspective through the patient journey analysis the team recognised that this could be very disruptive for patients and time consuming for staff. In response to this, they developed a 360 degree assessment of the patients that could be undertaken by any one of the professional groups, such as the physiotherapist, occupational therapist, and nurses working at the day Hospital. We introduced this concept of 360 degree assessment as a way of describing a holistic assessment of a patient by one individual. For example the nurses extended their assessments to included assessments previously undertaken by the physiotherapists and occupational therapists. The assessment includes physical assessment, which incorporates Ropers Activities of Daily Living (Roper 1990), medicine management, pain assessment, sensory loss assessment, continence, osteoporosis screening, 
psychological assessment, cognitive assessment, environmental, living arrangements and current levels of support. In order to implement this, a training programme was devised that enabled each professional group to develop more holistic assessment skills. Part of this included observing and being assessed on key assessment skills from the other professionals groups. As the team were in close proximity to each other the professional conducting the 360 degree assessment could always seek additional advice if required. Feedback from patients was very positive about this stating "I thought it was going to be very daunting with lots of people to see, I was really pleased I only had to see one person in the assessment" (patient feedback).

The advantage of the day hospital is that the holistic and multidisciplinary assessment and treatment of patients is under one roof. The patient will arrive in the day hospital and be seated in the day room. If they are there for assessment they will be called by the 360 practitioner and taken to a treatment room where the whole assessment takes place. If they are attending for follow up they will then be called by each professional that will treat them one at a time for 30 minute treatment slots. Usually the patient attends once a week. The initial assessment takes between one to two hours and a care plan is agreed. Patients then attend on a regular basis, for approximately six weeks, for interventions as identified by the care plan. The planned activities are defined by patient need and an individualised treatment plan is created for the patient. Where there is a need for trained professional intervention this is done by specialty. The care assistants however are trained to work across the disciplines and can where appropriate progress goals for more than one discipline.

The patients only attend for the duration of their appointment and transport wrapped around it so that they are not in the department for too long. They will sometimes have a 30 minute gap between appointments. This is to prevent fatigue and ensure they are maximising the input they are given.

These interventions can include goals around health promotion (such as smoking cessation, dietary advice, eye health and general fitness), balance and strengthening (developed in collaboration with the patients with specific exercise appropriate to their every day lives, and practical adaptations (such as equipment and techniques to maximise independence 
with ADL's). Other interventions will include psychological support around anxiety and depression management. There are targeted groups for education, exercises and support with specific conditions, such as Stroke and Parkinson's Disease. Also there are specialist clinics including Functional Electrical Stimulation (FES); management of vertigo with DixHallpike and Epley procedure. Suffice to say interventions are varied and specific to patient need.

\section{Better Together - the virtual ward}

The current drive towards integrated care within the NHS is the result of concerns raised regarding service fragmentation for patients; in particular a lack of coordination between primary, secondary and tertiary care (NHS 2014, Naylor et al 2015). In response to this, the local clinical commissioning group had prioritised improving integrated care specifically for patients with frailty and long term conditions, by holding regular interdisciplinary team meetings at General Practice (GP) surgeries to avoid unnecessary hospital admissions. Through the PDU process the day hospital team recognised that they could play a pivotal role in this, representing the acute trust at these new 'virtual ward rounds' (see case study in table 2). Patients who are over 65 years of age with complex needs related to their multipathology and are at risk or have had multiple hospital admissions are discussed. This can include those who have had a stroke and perhaps a cognitive impairment; or those at risk of falls and have airways disease, may well be seen. In essence patients who require more than one discipline to meet their needs, and would benefit Comprehensive Geriatric Assessment, are discussed with the MDT at these virtual ward rounds and if required are directly referred to the Day Hospital for assessment and treatment. These assessments can take place in the patients home if need be.

Table 2 Case Study of frail couple presented at a virtual ward meeting

At a virtual ward round one of the local GPs presented a case of an older couple with multiple complex medical conditions. In addition, to their complex medical needs there were complex social needs as they lived with their son who had learning difficulties and who was their main carer. The G.P was concerned that their physical health was deteriorating and the couple were at risk of hospital admission as the wife was refusing care support. 
It was decided that the anticipatory care team from the GP surgery would visit to complete a memory assessment and blood tests. Staff from the day hospital visited to undertake a home assessment of the families care needs as well as a falls risks assessment. As a result of the interdisciplinary day hospital assessment a memory gateway referral was made and equipment was provided to minimise the risk of falls. The team identified the wife's mood was very low and provided a baseline assessment to the GP, who was then able to initiative an appropriate plan of action. Finally a referral to social services (with the consent of the family) was made. This family continued to be monitored monthly at the virtual ward meetings avoiding the need to an unnecessary hospital admission.

The day hospital was ideally placed to support patients and families presented at the virtual ward meeting due to their interprofessional expertise they held. They are also able to fast track patients requiring interdisciplinary rehabilitation into the service to reduce the risk of avoidable hospital admissions. This has led to a more holistic, person centred approach to care through the day hospitals unique interface between primary and secondary care.

\section{Better Together - developing patient led service development}

Brown et al (2015) highlighted when considering the effectiveness of day hospitals, the views of those using those services must be taken into account. However the Kings Fund (2013) argues the potential peril of NHS providers using tokenistic mechanisms of collating patient feedback. As part of the PDU process the day hospital were really committed to ensuring clear engagement with patients and their carers in the redevelopment of their service and this was achieved in multiple ways. A 'You said we did' board was set up in the waiting area so that patients and their carers could see how the feedback they provided through the Friends and Family Test was being used by the staff at the day hospital. A day hospital newsletter was produced as a gateway informing patients of what was happening at the day hospital. This was supplemented by posters and flyers which were placed on tables through the day hospital, informing patients and their carers regarding the projects that were currently being undertaken encouraging the patients/carers to give their opinions 
on these as well as their experiences of using the service. As well as written communication, staff would regularly talk to the patient and their carers about the developments at the day hospital, this was both informally as well as formally via focus groups. Focus group sessions were held quarterly at the day hospital and patients and their carers were invited to come. The groups were led by an occupational therapist who is a member of the day hospital team. Those who have used the day hospital service within the previous two years are invited to attend. Some former patients attend alone, others with a member of their family. Some have been a member of the group for over two years, others may only attend for one meeting. The discussion in the focus groups may be initiated by feedback from the friends and family feedback, or service improvements are discussed with the group, or indeed initiated by the groups, this included creating a self-help list of 'Top 10 tips for future patients', and was written in collaboration with a student from the University. At the focus group the staff would then elicit feedback about different projects that were occurring as well as providing an opportunity for patients/carers to identify their own 'light bulb' moments which were then fed back into the PDU project plan.. One example of an idea brought to the focus group by a patient was to develop a 'Top Tips' sheet for health maintenance, things that they could do themselves to keep themselves fit and well. This was then developed by the team and brought back to the focus group to review. Also the members of the focus group reviewed written information given to patients attending the day hospital for the balance groups. The patients really valued this participation and many wanted to continue being part of it even when they left the service. When asked what motivated them to continue attending the focus groups the overwhelming response was of a connection to the service that helped them and wanting 'to give something back'.

\section{Better Together - developing a culture for proactive change}

Working through the PDU process has led to individual benefits for the practitioners working at the day hospital, and for patients accessing the services. From the outset the PDU leadership team wanted all staff and patients to shape the direction of the developments of the day hospital and have a shared vision that promoted a culture where everyone felt valued. Having a shared vision has been found to promote quality and safety improvement in patient care (Martin et al 2014), and this was the ultimate goal of the PDU process. A shared vision provided the direction for change and helped to inspire individuals 
as well as focus the energies of all team members. Care was taken to not overload the team with changes (Martin et al 2014) and a stepped approach was taken in implementing the many projects that were identified by the 'light bulbs'. Some changes were relatively simple to implement but remained patient centred. For example changing the timing of the fire alarm test maintained safety but reduced patient anxiety. The staff member suggesting the change felt appreciated that their contribution was acknowledged, implemented and therefore seen as worthwhile.

The PDU process and working with an academic facilitator provided a structure for the transformation of the culture of the unit, leading to a unit that was much more critically reflexive with a drive towards service improvement. Thus bridging the frequently referred to divide between theory and practice (Bonsaksen et al 2013; McQueen 2014). Not only did it provide staff with the confidence to share their work in the wider health field (Board et al 2016) it also enabled them to have a voice within their own organisation presenting the service to the board and having confidence in engaging with other local health and social care providers. This staff confidence was visible at all levels, and across all different staff groups (clinical, administrative, housekeeping) it has cemented that true service development can only be achieved through every member of the team actively working towards the common goal. Martin et al (2014) would reinforce this outcome of practice development finding that the process facilitates personal growth, confidence in practice expertise and greater autonomy.

The journey was not without its challenges. During the PDU process the Day Hospital was relocated to a new location, which has less physical space than before. Also some changes in team membership also occurred resulting in a slowing down in the changes being implemented. However, at the weekly meetings led by the clinical lead, staff would review their progress and set action plans together, either to accept the temporary slowing down in progress or set new targets, whatever was more appropriate. The team at the start of the process was already a collaborative cohesive team. However the PDU process reignited their energy and passion in providing new and innovative ways to meet the needs of the clients they cared for. The PDU process enabled the staff to recognise their contribution when 'every small change matters' and implementing these small changes was empowering. 
The ultimate goal at the start of the journey was to enhance the quality of care provided to patients in the day hospital. During the PDU process improvements in the triage and assessment process made by the team resulted in a reduction in waiting times despite a rise in demand for the service (Table 3). A more patient centred approach was introduced by the 360 degree assessment of patients by one professional (instead of three). This resulted in reducing patient anxiety as well as time to develop a good rapport and an improved depth of understanding of their needs. This has also led to a $30 \%$ reduction in the time it takes to complete the patient assessments resulting in an increased number of assessments being able to be completed from 20 to 29 per week, equating to an additional 468 assessments per year with no additional funding. Furthermore the virtual ward rounds have led to the introduction of home visits by the day hospital team ensuring a skilled timely service enabling the frail older person to remain at home for longer. The Family and Friends questionnaire is frequently completed and respondents are $100 \%$ satisfied with the service. However there is no room for complacency and the PDU tem continue to consider different ways to ensure the patients voice is well represented in the service delivery.

With regards to patient outcomes standardised measures are used to measure balance, mobility, cognition and mood at the initial assessment, and re measured at the end of the stay to demonstrate to patients the progress that they have made. At this point the team have not collated these formally to present efficacy of the service. This however is worth considering when demonstrating the effectiveness of the day hospital service. Other notable improvements in the service since the introduction of 360 degree assessments include improvements in patient flow with a throughput increase of $25 \%$ leading to waiting time reduction from 12 weeks to 5 weeks; also a reduction in the time to complete the same assessment by $30 \%$ and subsequently increasing the number of assessments available by 36 patients each month. Closer links with the University was also an additional outcome of the PDU process resulting in a matched funded PhD studentship position to investigate the effectiveness of the day hospital in supporting those older patients living with frailty. 
Table 3 Referral rate and waiting times since introduction of 360 degree assessment

\begin{tabular}{|l|l|l|}
\hline & Number of referrals & $\begin{array}{l}\text { Waiting time } \\
\text { (weeks) }\end{array}$ \\
\hline $\begin{array}{l}\text { December } \\
2015 \text { (before } \\
360 \text { degree } \\
\text { assessment } \\
\text { commenced) }\end{array}$ & 4.9 \\
\hline $\begin{array}{l}\text { October 2016 } \\
\text { (Following } \\
\text { introduction } \\
\text { of360 degree } \\
\text { assessment } \\
\text { Jan 2015) }\end{array}$ & 176 & 3.1 \\
\hline
\end{tabular}

\section{Conclusion}

The discourse around the needs of the frail older person is increasing. In addition, admission avoidance and enabling people to remain in their own homes has been influencing health and social care providers that have an ageing population. Further research into factors that influence the processes and outcomes to achieve effective integrated care for the frail older person is needed, and the impact these services have on reducing hospital admissions, or length of stay in acute hospitals. This paper has provided an example of the practice development undertaken by an interdisciplinary team at a day hospital. Undertaking a structured approach to their practice development the team have generated new ways of working resulting in a more patient centred focus as noted by their patients. Working collaboratively with internal and external partners the team have broadened the reach of their service, reducing their waiting times whilst their referrals increase. Furthermore the process highlights the contribution of a day hospital service that can smooth the transfer of care between primary and secondary services that puts the patients' needs first. 


\section{References}

Aliberti, M. J. et al. (2016) 'The Geriatric Day Hospital: Preliminary Data on an Innovative Model of Care in Brazil for Older Adults at Risk of Hospitalization', Journal- American Geriatrics Society, p. 2149.

Board M, Pigott L, Olive H. (2016) Symposium Better Together - A Day Hospitals Move Towards Integrated care. The British Society of Gerontology 45th Annual Conference, Stirling University Scotland, 06 Jul 2016 - 08 Jul 2016.

Bonsaksen, T, Celo, C, Myraunet, I, Granå, K, \& Ellingham, B (2013) 'Promoting academic-practice partnerships through students' practice placement', Int J Ther Rehabil, 20, 1, p. 33

British Geriatrics Society (2014) Fit for Frailty - consensus best practice guidance for the care of older people living in community and outpatient settings-a report from the British Geriatrics Society. London

Brown, L., A. Forster, J. Young, T et al (2015) Medical day hospital care for older people versus alternative forms of care: Cochrane Database of Systematic Reviews, p. 98.

Chartered Institute of Personnel and Development (2012) Leading culture change; employee engagement and public service transformation. London: Chartered Institute of Personnel and Development

Department for Environment, Food and Rural Affairs (DEFRA), (2011) Survey of public attitudes and behaviours towards the environment. Available from: https://data.gov.uk/dataset/survey of public attitudes and behaviours towards $t$ he environment

Hennessey, C, \& Fry, M (2016) 'Improving patient and staff outcomes using practice development', Int J Health Care Qual Assur ), 29, 8, pp. 853-863

King's Fund (2015) Transforming our health care system. London: The Kings Fund.

Kings Fund (2013) Patient-centred leadership - Rediscovering our purpose. London: The Kings Fund.

Martin, J, McCormack, B, Fitzsimons, D, and Spirig, R (2014) 'The importance of inspiring a shared vision', Int Practice Development Journal, 4, 2, pp. 1-15 
McCormack, B., Garbett, R. \& Manley, K (2004) A clearer vision of practice development. In: Practice Development in Nursing (eds B. McCormack, K. Manley \& R. Garbett), pp. 315-329. Blackwell Publishing Ltd., Oxford

McCormack, B, Manley, K, \& Titchen, A (2013) Practice development in nursing and healthcar, Chichester : Wiley-Blackwell

Mcqueen, J (2014) 'Bridging the research-practice gap: Are communities of practice the key? , Int J Ther Rehabil 21, 8, p. 358,

Naylor, C, Alderwick, $\mathrm{H}$ and Honeyman, M (2015) Acute hospitals and integrated care In: London: The King's Fund. Available from:

http://www.kingsfund.org.uk/sites/files/kf/field/field publication file/acutehospitals-and-integrated-care-march-2015.pdf.

NHS (2014) NHS England, Public Health England, Health Education England, Monitor, Care Quality Commission, NHS Trust Development Authority. Five Year Forward View. Available from https://www.england.nhs.uk/wpcontent/uploads/2014/10/5yfv-web.pdf [Accessed 26/1/17]

NICE (2015) Dementia, disability and frailty in later life - mid-life approaches to delay or prevent onset. NICE guideline [NG16] Available from https://www.nice.org.uk/guidance/ng16 Date accessed 31/10/17

Office of National Statistics (2015a) Ageing of the UK population. Available from http://webarchive.nationalarchives.gov.uk/20160105160709/http://www.ons.gov.u k/ons/rel/pop-estimate/population-estimates-for-uk--england-and-wales--scotlandand-northern-ireland/mid-2014/sty-ageing-of-the-uk-population.html

Office of National Statistics (2015b) Population Estimates for UK, England and Wales, Scotland and Northern Ireland: mid-2015 Available from https://www.ons.gov.uk/peoplepopulationandcommunity/populationandmigration/ populationestimates/bulletins/annualmidyearpopulationestimates/latest [Accessed 28/3/17] 
Oliver, D, Foot, C, and Humphries, R (2014) Making Our Health And Care Systems Fit For An Ageing Population, n.p.: London : The King's Fund.

Parker, S. G., P. Oliver, M. Pennington, J et al (2009) Rehabilitation of older patients: day hospital compared with rehabilitation at home. A randomised controlled trial: Health Technology Assessment (Winchester, England), 13, 1.

Roland, M, \& Paddison, C (2013) 'Better management of patients with multimorbidity', BMJ (Clinical Research Ed.), 346, p. f2510

Roper, N., Logan, W., Tierney, A., 1990. The Elements of Nursing (3rd edition). Edinburgh: Churchill Livingstone

World Health Organisation (2015) Aeing and Health fact sheet. Available from http://www.who.int/mediacentre/factsheets/fs404/en/ [accessed 6.9.17] 